\title{
Dynamic Spectrum Access Models: Toward an Engineering Perspective in the Spectrum Debate
}

\author{
Ömer Ileri and Narayan B. Mandayam, WINLAB - Rutgers University
}

\begin{abstract}
Until recently, the proponents of spectrum commons and the proponents of spectrum property rights had settled, like the armies of the Marne, into their entrenched positions, emerging only to launch periodic and unproductive attacks across a semantic and philosophical no man's land. Recently, however, there have been calls to move beyond these entrenched positions, by developing pragmatic models and solutions that capture some of the benefits of each philosophical position. In this article we cautiously enter that no man's land with two illustrative dynamic spectrum access models. While both of our models retain a bias toward usage of spectrum resources based on a spectrum property rights approach, they also promote dynamic access and short-term dedication of spectrum resources. We call these models dynamic property rights spectrum access (D-Pass) and dynamic commons property rights spectrum access (D-CPass). In this work we focus primarily on engineering issues, proposing the use of a spectrum policy server as a clearinghouse and specifying the spectrum access mechanisms relevant to each model. To demonstrate the useful studies enabled through these models, we present illustrative results via the bandwidth utilization achieved under each model. Our results indicate that both the spectrum access mechanism and market forces will play important roles in determining the resulting bandwidth utilization.
\end{abstract}

\section{The Spectrum Debate}

Efficient regimes for spectrum management have been a research focus since the earliest days of radio communications, but the mix of technologists, lawyers, and economists that has emerged in recent years has produced a new and lively debate. Unfortunately, the different approaches and languages of these researchers have made communication difficult, and no solid conclusions have emerged.

Traditional spectrum governance, long employed by the FCC, has had the goals of providing important services and protecting those important services from destructive interference. It has thus tended toward static long-term exclusivity of spectrum use in large geographic areas, often based on the radio technologies employed at the time of decision making. This has led to many successful applications like broadcasting and cellular, which can be cited as evidence by the proponents of spectrum property rights, but has also been criticized as inefficient in the overall use of spectrum. A recent report presenting statistics regarding spectrum utilization shows that even during the high demand period of a political convention such as the one held between August 31 and September 1, 2004 in New York City, only about 13 percent of the spectrum opportunities were utilized [1]. In addition to the static nature of such spectrum allocations, the inherent political and nonpolitical inefficiencies of government controllers also play a role in the poor spectrum utilization achieved [2].

The success of applications in the unlicensed bands (cordless telephony and WiFi being wellknown examples) has sparked a hot debate regarding how the spectrum governance employed by the FCC should be improved so that the new spectrum policy alleviates artificial spectrum scarcity, promotes efficiency, and also encourages innovation. However, this debate has very quickly gone past technical comparisons and has generated, as pointed out in [3], more passionate rhetoric than logic.

As mentioned above, the proposals for new governance regimes fall into two broad categories: spectrum property rights and spectrum commons. In its broadest sense, spectrum property rights refers to a governance mechanism in which portions of spectrum are owned by individuals (or companies). Such portions can be traded to other parties through monetary transactions, or used exclusively, in a flexible manner, with not many technical constraints. The spectrum commons approach, on the other hand, advocates that spectrum should be considered 


Another encouraging
development is the
agreement by some
parties from both
sides that the two
models are not polar
opposites, and there
may be governance
regimes that support
both exclusivity of
property rights and
the dynamic nature
of shared managed
access to a spectrum
commons.

Another encouraging common property, shared by all communicating parties, based on predefined but minimal rules or standards.

The spectrum property rights approach is motivated by the landmark work of $\mathrm{R}$. H. Coase [4], in which it is suggested that spectrum can be treated like land, and private ownership of spectrum is viable. The proponents of spectrum ownership believe that the spectrum should be allocated to the prospective spectrum holders through market forces. The spectrum holders would then have exclusive use of the spectrum portion they possess, without the potential of harmful interference from other parties. Alternatively, they would be able to trade their spectrum in a secondary market. The use of spectrum would be flexible, in that the authorized party could use the spectrum portion for any purpose. Thus, the focus in this approach is on transferring ownership of the spectrum from the government to private parties and substituting market forces for traditional spectrum regulation, overcoming two sources of inefficiency in the status quo regime. The common view is that since the 1990s the FCC has chosen a partial implementation of this approach by employing spectrum auctions as a means of licensing.

The spectrum commons approach, encouraged by the unlicensed spectrum band experiments, argues that as smart technologies evolve, communicating devices will become able to avoid interference through mutual cooperation and coexistence, and the spectrum will become unscarce. The emergence of cognitive and software defined radio concepts, multiple antenna and multicarrier techniques, as well as ultra wideband (UWB) technologies and mesh network topologies provide a technology panacea that proponents of this approach use to support their arguments. Communicating devices will be able to efficiently share a specified spectrum band through the enforcement of technical restrictions and multiple access protocols, without requiring exclusive access or private ownership. The analogy often articulated is that of a highway, which the motorists treat as a common property and can efficiently share as long as they abide by the traffic rules. The highway analogy also illustrates that in spite of all the smart radio technologies, there is still a need for a controller or enforcer. Thus, even the commons regime is a form of lightly controlled shared access [2].

Even though the generic descriptions of the two proposals seem clear, the lack of precise modeling creates many unanswered questions regarding the details of implementation. The exact nature of the controller or enforcer mechanisms in both models are but vaguely defined. The government's role in managing controlled access in a spectrum commons regime is not clear. This lack of clarity also pervades the many issues related to transferability and duration of transmission rights, transactions costs, and the specific mechanisms involved in allocating the spectrum when needed. It is not clear, for example, how often transmission rights are anticipated to change hands in a spectrum property rights model.

The ensuing gaps in the definitions, coupled with the inconsistent terminologies employed by the participating researchers, leads to confusion and miscommunication. A striking example would be the tragedy of taxonomy pointed out in [2], which refers to the apparent confusion between the terms open access regime and spectrum commons, particularly as encountered in the engineering communities. Open access regime, considered by some to be yet another alternative to the existing proposals for governance models, refers to a scheme where the spectrum is unowned, and access to spectrum is open to all with no limits or control at all. Thus, it is not the same as a spectrum commons. This confusion can partly be seen in discussions regarding the work of Noam [5], in which he proposes an open access scheme in which temporary (exclusive) spectrum access is granted to parties through congestion-based pricing. This work is often cited among those in favor of the spectrum commons approach, whereas many others argue that this approach cannot be classified under either the open access or spectrum commons model, and could in fact be considered a form of exclusive usage [6].

This lack of specific models involving the spectrum access/allocation and transaction techniques has led to vague and lengthy discussions that have not resolved the opposing views of these camps. The supporters of spectrum commons refer to the risk of monopolization and holdup, emphasizing that spectrum access should not be limited to those who can pay. Those who side with the property rights camp emphasize the risk referred to as the tragedy of commons, which predicts overuse and exploitation of common resources [7]. Political and philosophical arguments that relate to freedom of speech and the first amendment also find their place in this ongoing battle. Thus, what started as a technical challenge related to avoiding spectrum scarcity has turned into a passionate debate with political and philosophical overtones, and no clear path toward resolution. On the positive side, there have been recent calls (e.g., in [3, 7]) for the creation of specific spectrum access and management models for the above mentioned governance regimes, and detailed schemes and investigative tools that would permit both technical and political/philosophical comparisons of all such approaches.

Another encouraging development is the agreement by some parties from both sides that the two models are not polar opposites, and there may be governance regimes that support both exclusivity of property rights and the dynamic nature of shared managed access to a spectrum commons. Some hybrid schemes have been proposed, including end-state regulation and property rights with noninterfering easement. Endstate regulation is a regulatory scheme that contains bands dedicated for spectrum property rights governance along with other spectrum portions allocated for commons [7]. In property rights with noninterfering easement, the owner of any given spectrum portion (primary user) is supposed to permit secondary users to communicate in that band as long as they do not interfere with the transmissions of the primary user [7].

In this article we present two simple but realistic exemplifier models for specifying spectrum 
access and operator competition in a dynamic spectrum access setting. The dynamic spectrum access models considered rely on a quasi-centralized mechanism that coordinates spectrum sharing while retaining the distributed decision making of users. The framework here is enabled by the presence of a spectrum policy server [8-10], which functions as a controller/enforcer as well as a clearinghouse for spectrum allocations. In this framework there is a dynamic competition phase in which operators compete for users of spectrum. This phase is followed by a spectrum usage phase in which exclusive rights to spectrum are granted to operators and users. The access models we present are dynamic property-rights spectrum access (D-Pass) and dynamic-commons property-rights spectrum access (D-CPass). While both models promote exclusive use of spectrum resources, thus retaining a bias toward spectrum-property-rights-based usage, they also make use of dynamic access and short-term spectrum allocations. In both models operators compete with each other for customers through demand responsive pricing where users assert their preferences for the rates and prices offered by the operators [11]. Our emphasis is on presenting an engineering perspective toward developing practical models for use in the spectrum debate.

\section{MOdeling SPECTRUM MANAGEMENT IN DYNAMIC SPECtRUM ACCESS SetTINGS}

The dynamic spectrum access approach raises the issue of enabling architectures for coordinated spectrum access. In [8-10] this issue is addressed via the introduction of a spectrum policy server (SPS). The SPS is a central server responsible for coordinating spectrum access in a specified geographical region, as shown in Fig. 1. While the SPS, in a broad sense, can act as a broker for mediating spectrum access across heterogeneous systems and settings [12], the role of the SPS in this article will be coordinating dynamic spectrum access in a local interference region. In this sense the SPS's operation can be likened to that of the domain name server (DNS) or Dynamic Host Configuration Protocol (DHCP) in Internet engineering. We assume that the geographical boundaries of the interference region the SPS serves can be governed, for example, by either a signal strength threshold or a minimum throughput requirement the SPS can use to determine whether a given user is located in the region it serves. We assume that the spectrum resources are owned by the government, and portions of spectrum available in the interference region are leased on a temporary basis through the SPS, which acts as a clearinghouse.

We believe that with the advances in cognitive radio technologies, it will be possible for radios in a geographical region to identify and negotiate access to spectrum via the SPS for the serving area. Specifically, we assume that the SPS collects user-specific information upon entry of a user into the system and mediates operator interactions to form a basis for spectrum allocation deci-

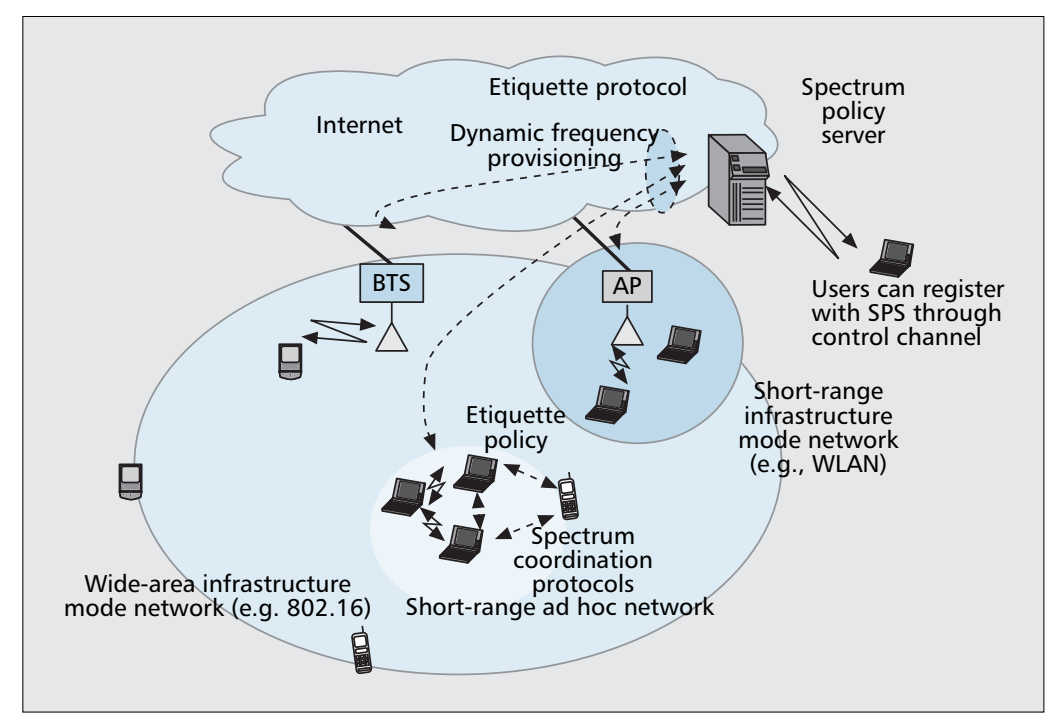

Figure 1. An examplary scenario of a spectrum policy server enabling dynamic spectrum access in heterogenous environments.

sions. User-specific information can be gathered from the user through any of several mechanisms, such as a control channel dedicated to establishing associations between users and the local SPS. The allocation decisions of the SPS could be based on maximization of any relevant prespecified criteria such as bandwidth utilization in the system, the sum rate achieved, or other system performance metrics. In this article we consider the spectrum allocation decisions of the SPS to be the result of maximization of the expected bandwidth utilization in the serving area.

In the D-Pass model, operators are allocated portions of spectrum by the SPS and compete for potential users (customers) given this allocation through demand responsive pricing, described in the next section. The operators pay for the amount of bandwidth they were assigned by the SPS whether or not they actually utilize all of it (spectrum ownership). The partition of the bandwidth is valid for a short-term duration that could be as short as a single communication session or possibly longer. Furthermore, no operator can use a portion of spectrum that is assigned to its competitors. In this sense the spectrum resource is considered under the framework of property rights with short-term dedication. The SPS determines the optimal partition of the available spectrum among the operators to maximize the expected bandwidth utilization in the system. The SPS mediates the operator competition through the realization of an iterative bidding scheme [9] reminiscent of a simultaneous ascending auction [13].

In the $D$-CPass model operators dynamically compete for spectrum as well as users through demand responsive pricing. Portions of spectrum are devoted to any operator that provides service to a user. The operators in return pay the SPS for the portion of spectrum they actually utilize. Operators compete for each user through an SPS-mediated iterative bidding scheme [8] that is reminiscent of a single-item ascending auction [13]. The result is that the SPS optimally partitions the total available bandwidth among differ- 


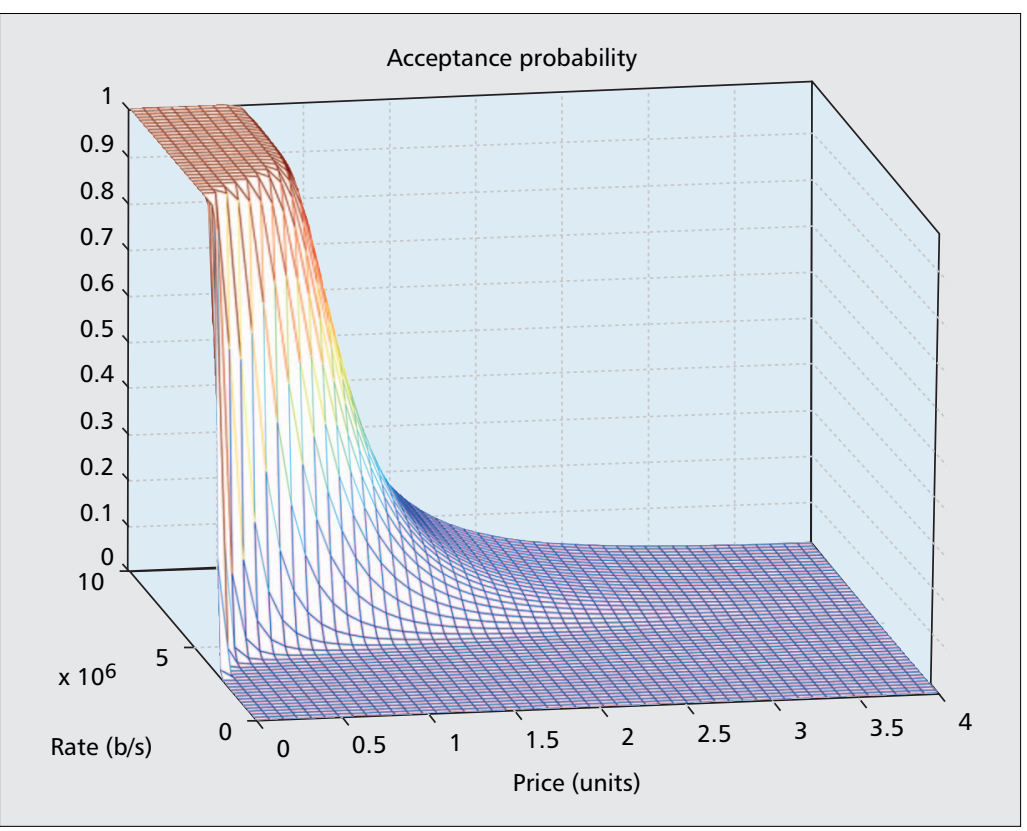

Figure 2. The acceptance probability is shown for $\mathrm{u}(\mathrm{R})=(\mathrm{R} / \mathrm{K})^{\mathrm{z}} / 1+$ $(\mathrm{R} / \mathrm{K})^{\mathrm{z}}$ with $\mathrm{K}=5 \times 106, \mathrm{z}=10, \mathrm{C}=1, \varepsilon=4$, and $\mu=4$.

ent user-operator sessions in order to maximize the expected bandwidth utilization. Note that during the competition phase there is no exclusivity, and all operators have access to all the available bandwidth even though the contention is still regulated by the SPS (suggesting a commons-like flavor). The spectrum usage is still exclusive; the operators transmit only in bandwidth portions allocated to users they serve.

It is important to emphasize that these schemes differ in two major ways in terms of spectrum management:

- In the D-CPass model all available spectrum is open to all operators during the bidding (competition) period, while in the D-Pass model operators have access only to the portion they are allocated individually.

- In the D-CPass model operators pay for bandwidth based on their usage, while in the $D$-Pass model operators pay for the portions allocated to them, whether or not they are actually able to utilize the whole spectrum portion.

\section{Demand Responsive Pricing}

Given that both models propose competitive spectrum allocation in which operators compete for users, it is important to model user appreciation for the service. The user's response to any operator's offered transmission rate $R[\mathrm{~b} / \mathrm{s}]$ with price asked $P$ [units] is modeled through an acceptance probability $A(R, P)$ that reflects its willingness to buy the offered service at the asked price. In both models, the operators try to attract any given user by inducing the highest acceptance probability from that user.

The operators are distinguished by the fact that they may have different service spectral efficiencies $r$ [b/s/Hz] and also different costs involved in serving any given user. The result is that each operator offers a specific transmission rate $R[\mathrm{~b} / \mathrm{s}]$ at a corresponding price $P$ [units] to each potential customer, where the offers are generated by the operator to maximize its expected profit. Note that the offered transmission rate $R$ for any given user, utilizes $R / r[\mathrm{~Hz}]$ of bandwidth. The expected profit is related to the associated $A(R, P)$ as well as the price asked $R$, the related fixed operational costs (independent of the offered rate $R$ ) and the payment of the operator to the SPS for the portion of spectrum utilized or allocated, depending on the model considered.

Intuitively, the acceptance probability $A(R, P)$ should be an increasing function of $R$ for fixed $P$ while decreasing in $P$ for fixed $R$. For illustration purposes, the acceptance probability model used in this work is the following [11]:

$$
A(R, P)=1-e^{-C u(R)^{\mu} P^{-\varepsilon}}
$$

where $\mu$ is the utility sensitivity of the user, $\varepsilon$ is the price sensitivity, and $C$ is an appropriate constant. $u(R)$ is a utility function for user satisfaction. Note that the above formulation provides a means to tune each user's preference. In the limiting special case when $A(R, P) \approx C R^{\mu} P^{-\varepsilon}$, acceptance probability is very similar to the Cobb-Douglas utility curves [14] that are used in economics to characterize the sensitivity to various inputs. A common example is characterizing the effects of inputs such as labor and capital on the production output. In our setting, the acceptance probability is the output that results as a function of the input parameters, namely the rate and price offers. Figure 2 illustrates the acceptance probability for a specified user as function of rate and price offers. Note that in the presence of multiple operators, we assume that each user only considers the service offer which invokes the greatest acceptance probability and rejects the rest.

\section{SPS-BASED DYNAMIC SPECTRUM ACCESS FOR MAXIMUM BANDWIDTH UTILIZATION}

A geographical serving area where the SPS has control of the available bandwidth $W_{A}$ is considered. A set of $M$ operators compete to provide services to an arbitrary set of $N$ users within the specified region. Each operator provides access to users through its base stations (access points) that are located in the serving area. The spectrum allocations determined by the SPS, as well as the service offers of the operators, are assumed to be valid for the whole duration of the communication sessions established between the users and operators.

The final spectrum allocation among the operators and users is the result of a hierarchical (two-tier) optimization process. Each spectrum allocation vector declared by the SPS in the upper tier induces operator competition in the lower tier, resulting in a set of rate and price offers as well as user acceptance probabilities. The SPS iteratively produces the allocation vector that maximizes the expected bandwidth utilization, which is defined as the sum of the individual bandwidth utilizations of each user 
weighted by its acceptance probability. Figure 3 illustrates this iterative optimization process.

\section{D-PASS MODEL}

In this model portions of the available spectrum $W_{A}$ are allocated to each operator. Given an allocation vector $\vec{W}=\left[W_{1} W_{2} \ldots W_{M}\right]^{T}$ for the $M$ operators as a result of the SPS maximizing the expected bandwidth utilization, operators compete simultaneously for $N$ users with rate and price offers (vectors). While making their vectoral offers, the operators are constrained not to exceed the bandwidth allocated to each, and try to maximize their expected profit and get each user to accept their service with the highest probability. The underlying operator competition results in an iterative bidding process reminiscent of a simultaneous ascending auction [13] where the bidding process is finalized when there are no new rate and price offers for any of the users (Fig. 4a). A detailed discussion of this mechanism and relevant implementation issues can be found in [9]. The SPS charges the operators for the amount of spectrum they are allocated, regardless of the extent of actual utilization. Each operator reserves the right to reject the bandwidth allocated to it at the beginning of the competition period (if it anticipates non-positive profit), thus staying out of operation in the short term.

Under this model, the expected profit for an operator $i$ is the sum of profits from all users it serves and is given as

$$
\begin{aligned}
& \backslash Q_{i}^{D P}(\vec{R}, \vec{P}) \\
& \quad=\sum_{n=1}^{N^{\prime}} A\left(R_{n}, P_{n}\right)\left(P_{n}-F_{i}\right)-W_{i} V, \quad i \in\{1, \ldots, M\}
\end{aligned}
$$

where $n$ is the user index for the users for which the specified operator is in a winning position, $W_{i}$ is the amount of bandwidth owned by the operator, $N^{\prime}$ is the number of users for which the operator outbids all other operators, and the other parameters are as defined before. $F_{i}$ [units] is the fixed operational cost incurred by the operator while serving any user; $V$ [units/Hz] is the price per unit bandwidth the SPS charges operator $i$.

It is important to note the difference between the fixed operational cost $F_{i}$ and sunk cost frequently encountered in pricing literature. The sunk cost refers to the type of cost incurred whether the service is provided or not. The fixed operational cost, on the other hand, is incurred only if the service is provided and does not depend on the quality of service. In the above formulation the sunk costs are not included; however, it is algebraically straightforward to show that inclusion of the sunk cost will result in similar expressions for the profit as given in Eq. 2 .

\section{D-CPASS MODEL}

In this model the SPS partitions the total available spectrum $W_{A}$ into $N$ nonoverlapping portions where $N$ is the number of users in the system. Given an allocation vector $\vec{W}=$ $\left[W_{1} W_{2} \ldots W_{N}\right]^{T}$ chosen by the SPS to maximize the expected bandwidth utilization, operators compete for each user independently through an iterative bidding process where they make rate

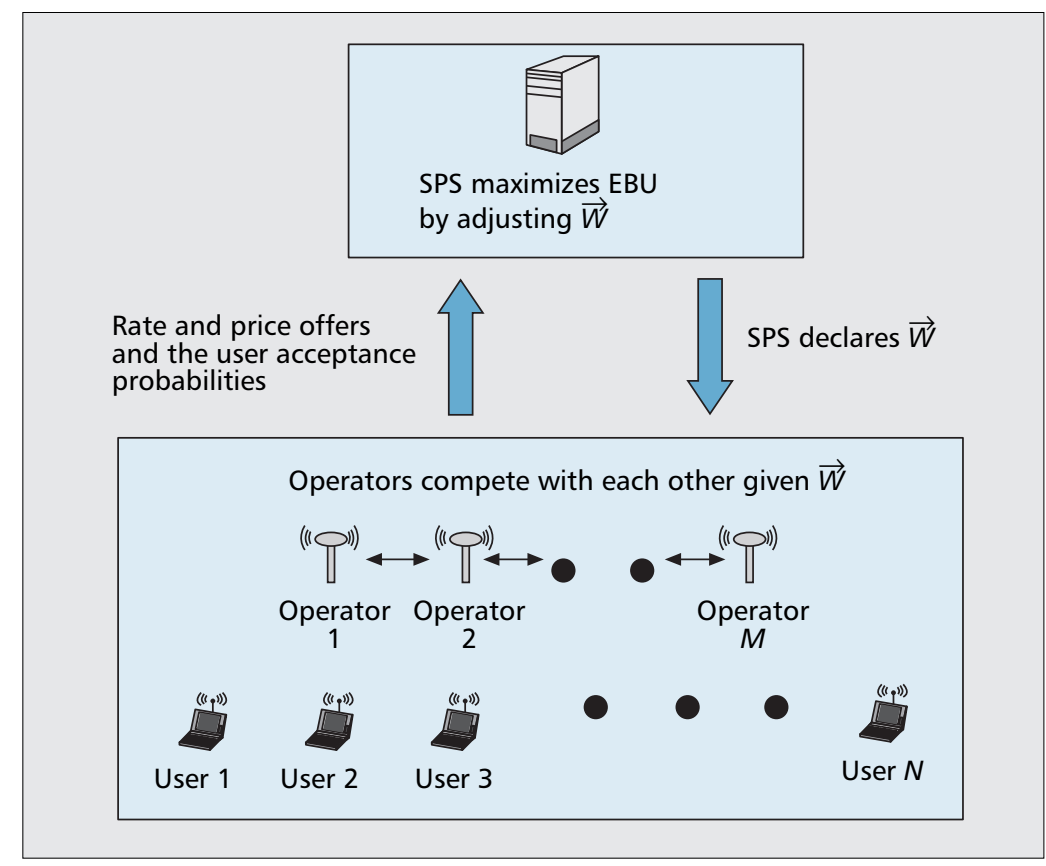

Figure 3. SPS-based hierarchical optimization for maximizing the expected bandwidth utilization (EBU).

and price offers to get each user to accept their service with the highest probability while also maximizing their expected profit. This process (Fig. 4b) is reminiscent of a single-item ascending bid auction [13] where the bidding process for any user is finalized when all but one of the operators is unable to make any rate and price offers with a higher acceptance probability while still achieving a non-negative profit [8]. During the competition phase, each operator competing for a specific user is subject to the constraint that it may not make rate offers that require bandwidths greater than the bandwidth allocated for the user in the allocation vector $\vec{W}$. The SPS charges the operators only for the exact amount of spectrum they use.

For each operator $i$, the expected profit to be achieved by serving any specified user $n$ through service offer $\left(R_{i, n}[\mathrm{~b} / \mathrm{s}], P_{i, n}\right.$ [units] $)$ is defined as

$$
\begin{aligned}
& Q_{i, n}^{D C P}\left(R_{i, n}, P_{i, n}\right) \\
& \quad=A\left(R_{i, n}, P_{i, n}\right)\left(P_{i, n}-F_{i}-V \times R_{i, n} / r_{i, n}\right), \\
& \quad i \in\{1, \ldots, M\}
\end{aligned}
$$

where $r_{i, n}[\mathrm{~b} / \mathrm{s} / \mathrm{Hz}]$ is the spectrum efficiency operator $i$ enjoys while serving the specified user. The last term $V \times R_{i, n} / r_{i, n}$ denotes the usage-based variable cost for the operator.

\section{Model Evaluations}

For the purpose of illustration, we present numerical results that correspond to a simple linear geographical region with $M=2$ operators and $N=5$ users. The system and access point (AP) locations are depicted in Fig. 5, where an instantiation of the five user locations is shown. We assume that both operators use the same technology and have one AP each in the serving area of interest.

Note that the fixed operational $\cos t F_{i}$ for 


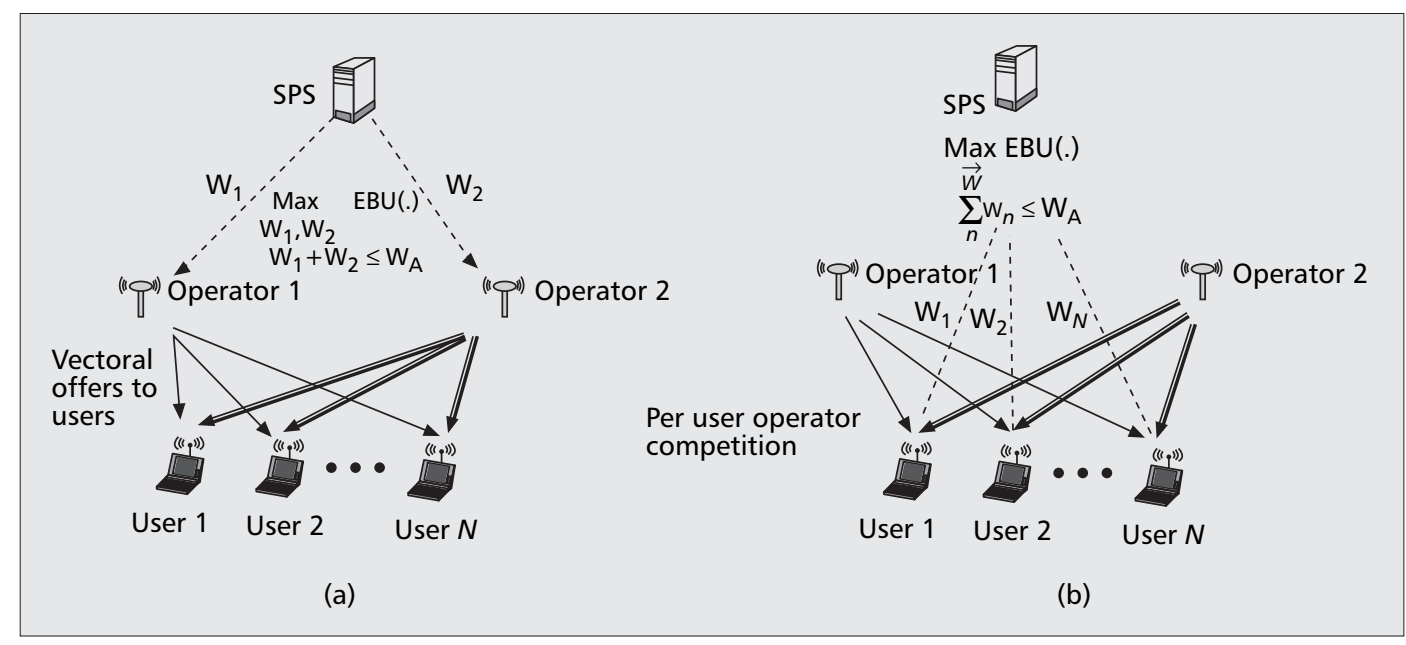

Figure 4. SPS-based spectrum allocation for a two-operator system in a) D-Pass model - SPS only partitions the bandwidth among operators who in turn compete simultaneously for the users; $b$ ) D-CPass model - SPS mediates iterative bidding processes among operators independently for each user.

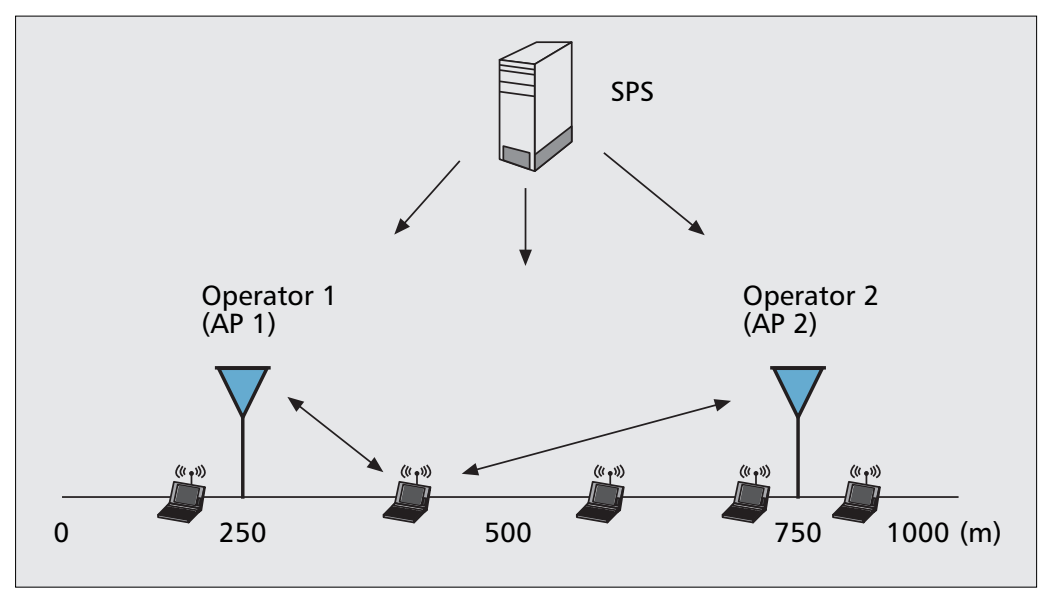

Figure 5. Geographical region with two operators.

operator $i$ is an involved function of many economic, social, and technological parameters, and many sophisticated cost models can be developed for it [13]. However, for simplicity of illustration, we consider a symmetric situation where the fixed operational $\operatorname{cost} F_{i}$ is identical for each operator: $F_{1}=F_{2}=F$.

The spectral efficiency between an AP $k$ and the user's mobile terminal is determined as

$$
r_{k}=\log _{2}\left[1+\frac{P_{s}}{N_{o}}\left(\frac{d_{k}}{L / 4}\right)^{-2}\right],
$$

where $P_{s}$ is the signal power, $N_{o}$ is the additive white Gaussian noise (AWGN) variance, $d_{k}$ is the distance between AP $k$ and the terminal, and $L$ is the total length of the linear region in Fig. 5 $(L=1000 \mathrm{~m})$. We set $P_{s}=2 N_{o}$, which guarantees a signal-to-noise ratio (SNR) of $3 \mathrm{~dB}$ at the distance of $L / 4=250 \mathrm{~m}$ from the base station.

The available bandwidth is $W_{A}=10 \mathrm{MHz}$, and the user acceptance probability and corresponding parameters are selected as in Fig. 5. In order to keep the spectrum allocation problems tractable in both models, the bandwidth is quantized to be made of basic units approximately $380 \mathrm{kHz}$ wide.
We parameterize the results in the fixed operational cost $F$ [units] and the unit variable cost $V$ [units/Hz]. For each tested $(F, V)$ pair, 300 different instantiations of the user locations are tested by randomly locating five users along the linear region, assuming uniform distribution of user locations. The results are then averaged over all 300 different instantiations, and the curves shown refer to the resulting average values achieved.

Figure 6a shows the expected bandwidth utilizations achieved in both models (D-Pass and DCPass) as a function of $F+V W_{A}$ for a specific ratio $V W_{A} / F=4$. Figure $6 \mathrm{~b}$ shows the performance comparison for the two optimum allocation models when $V=0$ (i.e., spectrum usage is free).

Figure 6a suggests that for values of $F$ and $V$ sufficiently close to zero (negligible costs), both models achieve similar spectrum utilizations in this example. It is also observed that the expected bandwidth utilization is decreasing in both models with increasing costs. As the values of $F$ and $V$ become non-negligible, the D-Pass model initially starts to achieve greater expected bandwidth utilization as opposed to the D-CPass model. However, when the cost pair $(F, V)$ grow much higher, this trend is reversed, and the DCPass model performs better. When $V=0$ (Fig. $6 \mathrm{~b})$, it is observed that the D-CPass model always has superior performance.

We now present an interpretation of the above trends in relation to the two models considered. In both models there are factors related to dynamic access as well as market forces (cost and payment mechanisms) that affect the bandwidth utilization achieved. In the D-Pass model only part of the spectrum is available to each operator, which can cause inefficiencies. However, the SPS-enforced allocation mechanism requires the operators to precompetitively invest in spectrum portions. Thus, this mechanism induces greater incentives for the operator to make attractive rate offers to users, thereby increasing bandwidth utilization. This explains the initial improvement in bandwidth utilization in the D-Pass scheme with increasing costs. However, when the costs become much higher, the SPS is unable to produce allocation vectors affordable to the operators. In this 


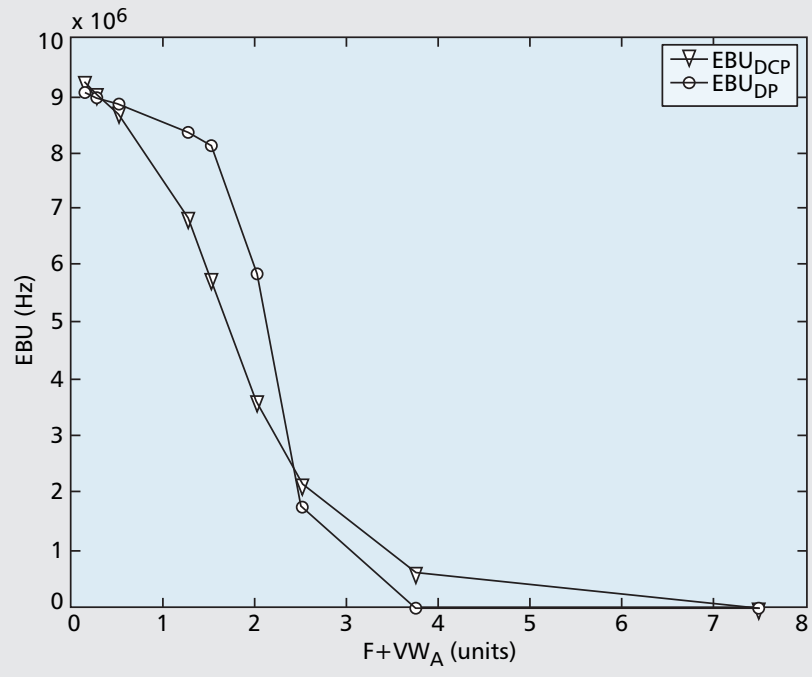

(a)

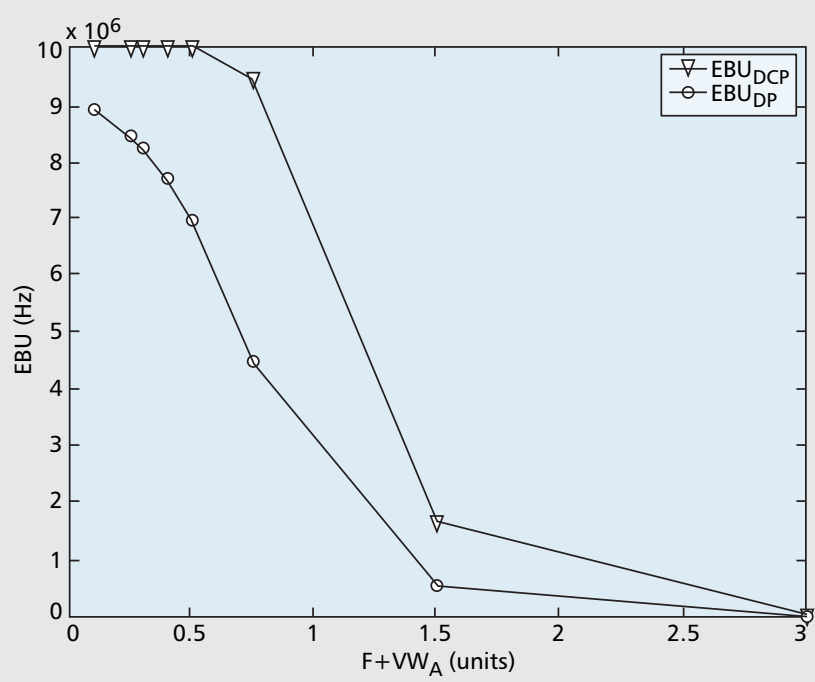

(b)

Figure 6. Performance comparisons between D-Pass and D-CPass models: a) expected bandwidth utilization (EBU) in D-CPass and $D$-Pass models for $V W_{A} / F=4$ and $W_{A}=107 \mathrm{~Hz}$; b) EBU in D-CPass and D-Pass models for $V=0$ and $W_{A}=107 \mathrm{~Hz}$.

case, since the payment for the spectrum is due regardless of the utilization (Eq. 2), the operators are unable to make rate and price offers that are attractive to users, resulting in reduced values of the induced acceptance probability. This results in the operators opting out in the short term, thereby reducing bandwidth utilization below that of the D-CPass model. Note that the payment mechanism in the D-CPass model does not require precompetitive payments, and operators pay for the part of spectrum they actually use (Eq. 3). The operators have access to all available bandwidth during the competition phase and determine the amount of bandwidth they purchase for any given user. Thus, the above mentioned market forces relevant to the D-Pass model are not present in the D-CPass model. Note that when $V=0$, the above mentioned dynamics regarding the precompetition investments in the D-Pass model are not valid, since spectrum portions are allocated for free. Thus, the D-CPass model always achieves better performance when $V=0$ since both operators have commons-like access to the entire spectrum during the competition phase. Recall that usage still remains exclusive similar to the property rights regime.

These results demonstrate that in addition to the specific spectrum access mechanism, the market forces employed and relevant payment schemes in the models have an important role in determining the achieved performance. Furthermore, the specific numerical results shown here are functions of cost models and the width of the basic spectrum units.

\section{CONCLUSIONS AND FUTURE DIRECTIONS}

Inspired by the spectrum debate, we have taken an engineering perspective toward developing dynamic spectrum access models and illustrate this approach with two such models, D-CPass and D-Pass. While retaining a bias toward the spectrum property rights approach, these models also promote dynamic access and short-term dedication of spectrum resources. These models rely on a quasi-centralized mechanism that coordinates spectrum sharing while retaining the distributed decision making of users. To accomplish this, we have proposed the use of a spectrum policy server to function as a controller/enforcer as well as a clearinghouse to mediate market-based spectrum allocation. We have presented illustrative results which indicate that both the spectrum access mechanism and market forces play important roles in the resulting bandwidth utilization. We believe that the simple yet exemplary models presented in this article provide a foundation for more realistic engineering models that can shape spectrum policy.

There are several interesting research issues to be addressed in the framework introduced in this article. An important one would be to devise ways to successfully convey user-specific information $(A(R, P))$ from users to the SPS. It might not always be possible for the user to transmit a detailed acceptance probability profile to the SPS. This may be due to intermittent connectivity, and/or security and privacy concerns. In such scenarios designing robust spectrum allocation mechanisms poses several interesting technical challenges [15]. Yet another interesting research challenge would be to devise simplified optimization algorithms for the SPS, given that the SPS is responsible for multidimensional optimizations of objective functions that may be neither concave nor convex. In such scenarios clustering algorithms, in which users are clustered into groups and spectrum allocations are rendered for such groups, provide interesting engineered solution approaches [15].

\section{ACKNOWLEDGEMENTS}

The authors would like to thank to the editor and the reviewers for their insightful comments. The authors would also like to thank Dragan 
There are several interesting research

issues to be addressed in the

framework introduced in this

article. An important one among them would be to devise ways to successfully convey user specific information $(A(R, P))$ from the users to the SPS.
Samardzija of Bell Labs, Alcatel-Lucent Technologies, and Richard Frenkiel of Winlab Rutgers University for their valuable contributions in various phases of the work presented in this article. This work is supported in part by the NSF under grant number NeTS-0434854 and the Defense Spectrum Office (DSO) of the Defense Information Systems Agency.

\section{REFERENCES}

[1] M. McHenry and D. McCloskey," New York City Spectrum Occupancy Measurements September 2004," tech. rep., Dec. 2004.

[2] J. Brito, "The Spectrum Commons in Theory and Practice," working paper in regulatory studies, 2006.

[3] J. M. Peha, "Approaches to Spectrum Sharing," IEEE Commun. Mag., vol. 43, no. 2, Feb. 2005, pp. 10-12.

[4] R. H. Coase, "The Federal Communications Commission," J. Law and Economics, vol. 2, Oct. 1959, pp. 1-40.

[5] E. Noam, "Spectrum Auctions: Yesterday's Heresy, Today's Orthodoxy, Tomorrow's Anachronism. Taking the Next Step to Open Spectrum Access," J. Law and Economics, vol. 41, no. 2, Oct. 1998, pp. 765-90.

[6] T. Hazlett, "Spectrum Flash Dance: Eli Noam's Proposal for "Open Access" to Radio Waves," J. Law and Economics, vol. 41, no. 2, Oct. 1998, pp. 805-20.

[7] G. R. Faulhaber, "The Question of Spectrum: Technology, Management and Regime Change," Conf. Economics, Technology and Policy of Unlicensed Spectrum, East Lansing, MI, 2005.

[8] O. Ileri et al., "Demand Responsive Pricing and Competitive Spectrum Allocation via a Spectrum Server," Proc. IEEE DySpan, Baltimore, MD, 8-11 Nov., 2005, pp. 194-202.

[9] O. Ileri, D. Samardzija, and N. B. Mandayam, "Dynamic Property Rights Spectrum Access: Flexible Ownership Based Spectrum Management," Proc. IEEE DySpan, Dublin, Ireland, 17-20 Apr., 2007.

[10] C. C. Raman, R. Yates, and N.B. Mandayam, "Scheduling Variable Rate Links via a Spectrum Server," Proc. IEEE DySpan, Baltimore, MD, 8-11 Nov., 2005, pp. 110-18.

[11] L. Badia et al., "Demand and Pricing Effects on the Radio Resource Allocation of Multimedia Communication Systems," Proc. IEEE GLOBECOM, San Francisco, CA, Dec. 2003.

[12] M. M. Buddhikot et al., "Dimsumnet: New Directions in Wireless Networking Using Coordinated Dynamic Spectrum Access," Proc. IEEE WoWMoM '05, 13-16 June 2005, pp. 78-85.

[13] C. Courcoubetis and R. Weber, Pricing Communication Networks, Wiley, 2003.

[14] H. R. Varian, Intermediate Microeconomics: A Modern Approach, W. W. Norton, 1987.
[15] O. Ileri, "Dynamic Spectrum Access Models: Towards an Engineering Perspective in the Spectrum Debate," Ph.D. dissertation, Rutgers Univ., New Brunswick, NJ، Oct. 2007

\section{BIOGRAPHIES}

NARAYAN MANDAYAM (narayan@winlab.rutgers.edu) received a B.Tech (Hons.) degree in 1989 from the Indian Institute of Technology, Kharagpur, and M.S. and Ph.D. degrees in 1991 and 1994 from Rice University, Houston, Texas, all in electrical engineering. From 1994 to 1996 he was a research associate at the Wireless Information Network Laboratory (WINLAB), Department of Electrical and Computer Engineering, Rutgers University. In September 1996 he joined the faculty of the Electrical and Computer Department at Rutgers where he became an associate professor in 2001 and a professor in 2003. Currently, he also serves as associate director at WINLAB. He was a visiting faculty fellow in the Department of Electrical Engineering, Princeton University in fall 2002 and a visiting faculty at the Indian Institute of Science in spring 2003. His research interests are in various aspects of wireless data transmission and radio resource management with emphasis on techniques for cognitive radio technologies. He is a recipient of the Institute Silver Medal from the Indian Institute of Technology, Kharagpur in 1989 and the National Science Foundation CAREER Award in 1998. He was selected by the National Academy of Engineering in 1999 for the Annual Symposium on Frontiers of Engineering. He is a coauthor with C. Comaniciu and H. V. Poor of the book Wireless Networks: Multiuser Detection in Cross-Layer Design (Springer). He has served as an Editor for IEEE Communication Letters (1999-2002) and IEEE Transactions on Wireless Communications (2002-2004). He has also served as a guest editor of the IEEE JSAC Special Issue on Adaptive, Spectrum Agile and Cognitive Radio Networks (April, 2007). He is currently serving as a guest editor of the upcoming IEEE JSAC Special Issue on Game Theory in Communication Systems.

ÖMER ILERI (omer.ileri@ieee.org) received a B.S. degree (honors) in electrical/electronic engineering from Bogaziçi University, Istanbul, Turkey, in 2001, and M.S. and Ph.D. degrees in electrical and computer engineering from Rutgers University, New Brunswick, New Jersey, in 2003 and 2007, respectively. During his Ph.D. studies, he was with the Wireless Information Network Laboratory (WINLAB), Rutgers University. He was a summer intern in Bell Laboratories, Lucent Technologies, Holmdel, New Jersey, in 2005. $\mathrm{He}$ is currently at the Radio Communications Laboratory at the Department of Communication Systems at KTH, Sweden, as a post-doctoral research fellow. His research interests lie in the broad areas of wireless networks and communications, radio resource management, and communication networks economics. 